\title{
Relativistic descriptions of final-state interactions in charged-current neutrino-nucleus scattering at ArgoNeuT kinematics
}

\author{
Andrea Meucci, Carlotta Giusti, and Matteo Vorabbi \\ Dipartimento di Fisica, Università degli Studi di Pavia and \\ INFN, Sezione di Pavia, via A. Bassi 6, I-27100 Pavia, Italy
}

(Dated: July 7, 2018)

\begin{abstract}
The analysis of the recent charged-current neutrino-nucleus scattering cross sections measured by the ArgoNeuT Collaboration requires relativistic theoretical descriptions also accounting for the role of final-state interactions. In this work, we evaluate differential neutrino-nucleus cross sections with the relativistic Green's function model, where final-state interactions are described in the inclusive scattering consistently with the exclusive scattering using a complex optical potential. The sensitivity to the parameterization adopted for the phenomenological optical potential is discussed. The predictions of the relativistic Green's function model are compared with the results of different descriptions of final-state interactions.
\end{abstract}

PACS numbers: 25.30.Pt; 13.15.+g; 24.10.Jv

Keywords: Neutrino scattering; Neutrino-induced reactions; Relativistic models

\section{INTRODUCTION}

In the past decade many neutrino oscillation results have been presented by different collaborations [1 15], and a phenomenological extension of the Standard Model has been proposed that involves three neutrino mass states, over which the three flavors of neutrinos are distributed. Despite its successful predictions, this can be considered as an extension of the Standard Model that does not address fundamental questions, e.g., small masses and large mixing angles compared to quark sector, and has raised a large debate over other possible unexpected properties of neutrinos that could lead to a more complete understanding of neutrino physics [16 21]. To gain a deeper understanding of neutrino phenomenology the reduction of uncertainties in baseline neutrino oscillation experiments is mandatory.

Because of the interest in oscillation measurements, in recent years various neutrino-nucleus differential cross sections have been presented 22 26] and are planned in the near future [27-29]. Differential cross sections are important to obtain a complete kinematical determination of neutrino-nucleus scattering and a clear understanding of neutrino-nucleus reactions is crucial for the analysis of experimental measurements.

The ArgoNeuT Collaboration has recently reported 26] a measurement of the muon neutrino chargedcurrent (CC) flux-averaged differential cross section on ${ }^{40} \mathrm{Ar}$ in an energy range up to $50 \mathrm{GeV}$. A liquid Argon detector is very interesting because it has excellent potentialities to make precise measurements of a very large class of neutrino interactions from the MeV energy scale to multi-GeV events [30]. The ArgoNeuT measurement has proven the validity of this experimental technique and, hoperfully, new data will be available in the future. For instance, a calculation of neutrino capture cross sections for solar neutrinos that could be addressed by this new generation of detectors is presented in [31].

The energy region considered in the ArgoNeuT exper- iment, with average neutrino energy of $\left\langle E_{\nu}\right\rangle=4.3 \mathrm{GeV}$, requires the use of a relativistic model, where not only relativistic kinematics is considered, but also nuclear dynamics and current operators are described within a relativistic framework.

The first measurement of the charged-current quasielastic (CCQE) flux-averaged double-differential muon neutrino cross section on ${ }^{12} \mathrm{C}$ by the MiniBooNE Collaboration [22] has raised extensive discussions. In particular, the experimental cross section is usually underestimated by the relativistic Fermi gas model and by other more sophisticated models based on the impulse approximation [32 36], unless the nucleon axial mass $M_{A}$ is significantly enlarged with respect to the world average value of $1.03 \mathrm{GeV} / c^{2}$. It is reasonable to assume that the larger axial mass obtained from the MiniBooNE data on ${ }^{12} \mathrm{C}$ can be interpreted as an effective way to include medium effects that are not taken into account by the models; this is another indication that a precise knowledge of lepton-nucleus cross sections, where uncertainties on nuclear effects are reduced as much as possible, is necessary. Moreover, any model aimed to describe neutrino-nucles scattering should first be tested against electron scattering data in the same kinematic region.

At intermediate energy, quasielastic (QE) electron scattering calculations [37, 38], which were able to successfully describe a wide number of experimental data, can provide a useful tool to study neutrino-induced processes. There are, however, indications that the reaction can have significant contributions from effects beyond the impulse approximation (IA) in some energy regions where the neutrino flux has still significant strength. For instance, in the models of 39 44] the contribution of multinucleon excitations to CCQE scattering has been found sizable and able to bring the theory in agreement with the experimental MiniBooNE cross sections without increasing the value of $M_{A}$. The role of processes involving two-body currents compared to the IA models 
has been discussed in [33, 45, 49]. A careful evaluation of all nuclear effects and of the relevance of multinucleon emission and of some non-nucleonic contributions 50 53 would be, without a doubt, useful for a deeper understanding of the reaction dynamics.

The relevance of final state interactions (FSI) has been clearly stated for the exclusive $\left(e, e^{\prime} p\right)$ reaction, where the use of a complex optical potential (OP) in the distorted-wave impulse approximation (DWIA) is required [37, 38, 54 59]. The imaginary part of the OP produces an absorption that reduces the cross section and accounts for the loss of part of the incident flux in the elastically scattered beam to the inelastic channels which are open. In the inclusive scattering only the emitted lepton is detected, the final nuclear state is not determined and all elastic and inelastic channels contribute. Thus, a different treatment of FSI is required, where all finalstate channels are retained and the total flux, although redistributed among all possible channels, is conserved.

Different approaches have been used to describe FSI in relativistic calculations for the inclusive QE electronand neutrino-nucleus scattering $[60-72]$. In the relativistic plane-wave impulse approximation (RPWIA), FSI are simply neglected. In other approaches FSI are included in DWIA calculations where the final nucleon state is evaluated with real potentials, either retaining only the real part of the relativistic energy-dependent complex optical potential (rROP), or using the same relativistic mean field potential considered in describing the initial nucleon state (RMF). Although conserving the flux, the rROP is unsatisfactory from a theoretical point of view. On the contrary, the RMF, where the same strong energyindependent real potential is used for both bound and scattering states, fulfills the dispersion relations 73 and also the continuity equation.

In a different description of FSI relativistic Green's function (RGF) techniques [63, 64, 70, 71, 74 78] are used. In the RGF model, under suitable approximations, which are basically related to the IA, the components of the nuclear response are written in terms of the single particle optical model Green's function; its spectral representation, that is based on a biorthogonal expansion in terms of a non-Hermitian optical potential $\mathcal{H}$ and of its Hermitian conjugate $\mathcal{H}^{\dagger}$, can be exploited to avoid the explicit calculation of the single particle Green's function and obtain the components of the hadron tensor [63, 64]. Calculations require matrix elements of the same type as the DWIA ones of the exclusive $\left(e, e^{\prime} p\right)$ process in [55], but involve eigenfunctions of both $\mathcal{H}$ and $\mathcal{H}^{\dagger}$, where the imaginary part has an opposite sign and gives in one case a loss and in the other case a gain of strength. The RGF formalism allows us to reconstruct the flux lost into nonelastic channels in the case of the inclusive response starting from the complex optical potential which describes elastic nucleon-nucleus scattering data and to include contributions which are not included in the RMF and in other models based on the IA. Moreover, with the use of the same complex optical potential, it provides a

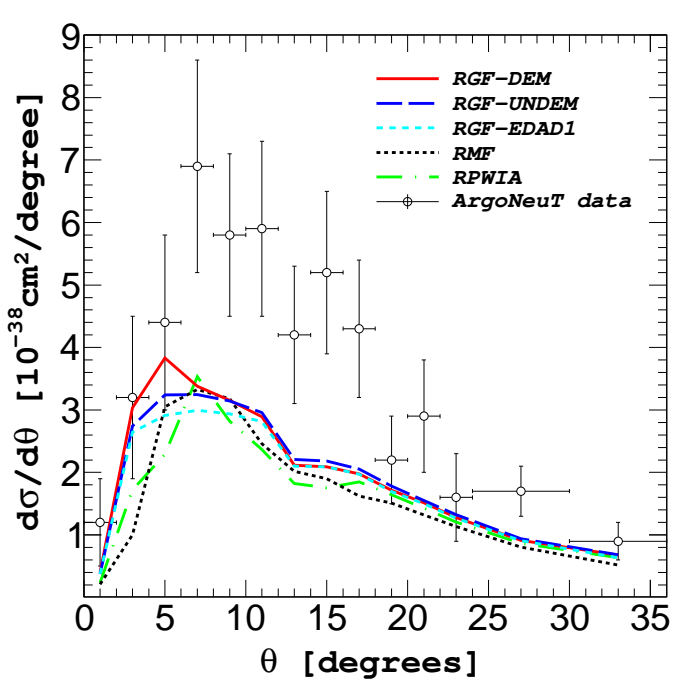

Figure 1. (Color online) Flux-averaged differential cross section $d \sigma / d \theta$ for the reaction ${ }^{40} \operatorname{Ar}\left(\nu_{\mu}, \mu^{-}\right)$as a function of the muon scattering angle $\theta$. The data are from ArgoNeuT [26].

consistent treatment of FSI in the exclusive and in the inclusive scattering. In addition, because of the analyticity properties of the optical potential, it fulfills the Coulomb sum rule 64, 73, 74].

These different descriptions of FSI have been compared in [70] for the inclusive QE electron scattering, in [71] for the CCQE neutrino scattering, and in [79 81] with the CCQE and NCE MiniBooNE data. Both RMF and RGF are able to describe the shape of the CCQE experimental data, only the RGF gives cross sections of the same magnitude as the experimental ones without the need to increase the value of $M_{A}[79,80]$. Similar results are obtained in [81], where the RGF results and their interpretation in comparison with the $\mathrm{NCE}$ data from MiniBooNE are discussed.

In this paper the results of different relativistic descriptions of FSI for CC $\nu$-nucleus scattering are presented and discussed for the differential cross section averaged over the $\nu_{\mu}$ ArgoNeuT flux. We are aware of the interpretative questions that may be connected to the use of models developed for the $\mathrm{QE}$ regime in a kinematic situation, with the $\nu_{\mu}$ ArgoNeuT flux up to $50 \mathrm{GeV}$, where other processes beyond the IA, which are not included in the models considered here, can give significant contributions. Nevertheless we believe that our calculations can give useful information about the role of nuclear effects in the analysis of $\nu_{\mu}-{ }^{40} \mathrm{Ar}$ scattering and about the uncertainties which are related to their evaluation.

\section{RESULTS AND DISCUSSION}

In all the calculations presented in this work the bound nucleon states are taken as self-consistent Dirac-Hartree solutions derived within a relativistic mean field approach 


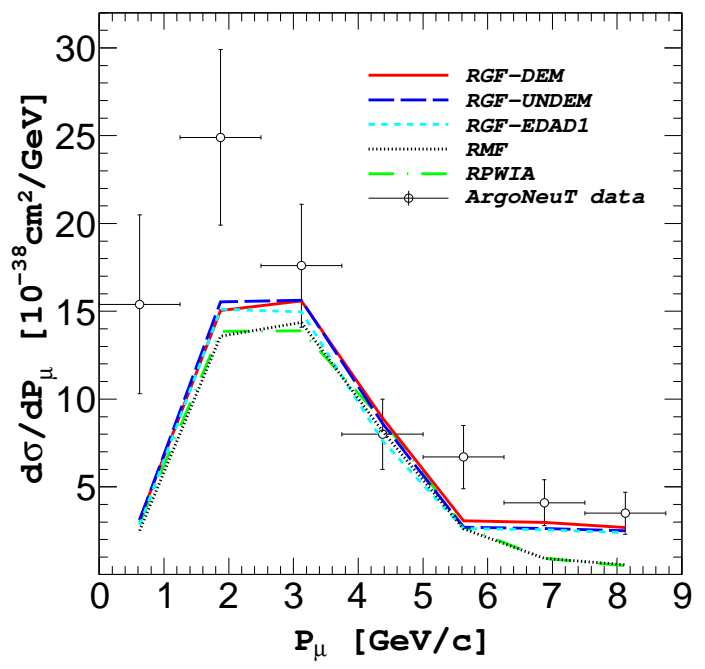

Figure 2. (Color online) Flux-averaged differential cross section $d \sigma / d P_{\mu}$ for the reaction ${ }^{40} \operatorname{Ar}\left(\nu_{\mu}, \mu^{-}\right)$as a function of the muon momentum $P_{\mu}$. The data are from ArgoNeuT [26].

using a Lagrangian containing $\sigma, \omega$, and $\rho$ mesons [82], with medium dependent parameterizations of the mesonnucleon vertices that can be more directly related to the underlying microscopic description of nuclear interactions [83 85]. The same relativistic mean field approach has been used to calculate the bound state wave functions in [78, 86, 87], where the cross sections of the exclusive quasi-free $\left(e, e^{\prime} p\right)$ and inclusive $\mathrm{QE}\left(e, e^{\prime}\right)$ reactions have been presented and discussed for oxygen and calcium isotopic chains.

In the RGF calculations we have used three parameterizations for the relativistic OP of ${ }^{40} \mathrm{Ar}$ : the EnergyDependent and A-Dependent EDAD1 (where the $E$ represents the energy and the $A$ the atomic number) OP of [88] and the more recent Democratic (DEM) and the Undemocratic (UNDEM) phenomenological OPs of 89]. We note that all these three parameterizations are global ones, since they are obtained through a fit to elastic proton-scattering data on a wide range of nuclei and, as such, they depend on the atomic number $A$ and are not constructed to reproduce the ${ }^{40} \mathrm{Ar}$ phenomenology.

In Fig. 1 the CC differential cross section $d \sigma / d \theta$ integrated over the ArgoNeuT flux is shown as a function of the muon scattering angle $\theta$. All the calculations give results in reasonable agreement with the experimental shape but generally underpredict the magnitude of the experimental cross section. We note that in the RPWIA FSI are completely neglected, while in the RMF the same strong energy-independent real potential is used for bound and scattering states. The comparison between the RGF results obtained with the EDAD1, DEM, and UNDEM potentials can give an idea of how the predictions of the model are affected by uncertainties in the determination of the phenomenological OP. The differences depend on the energy and momentum transfer and
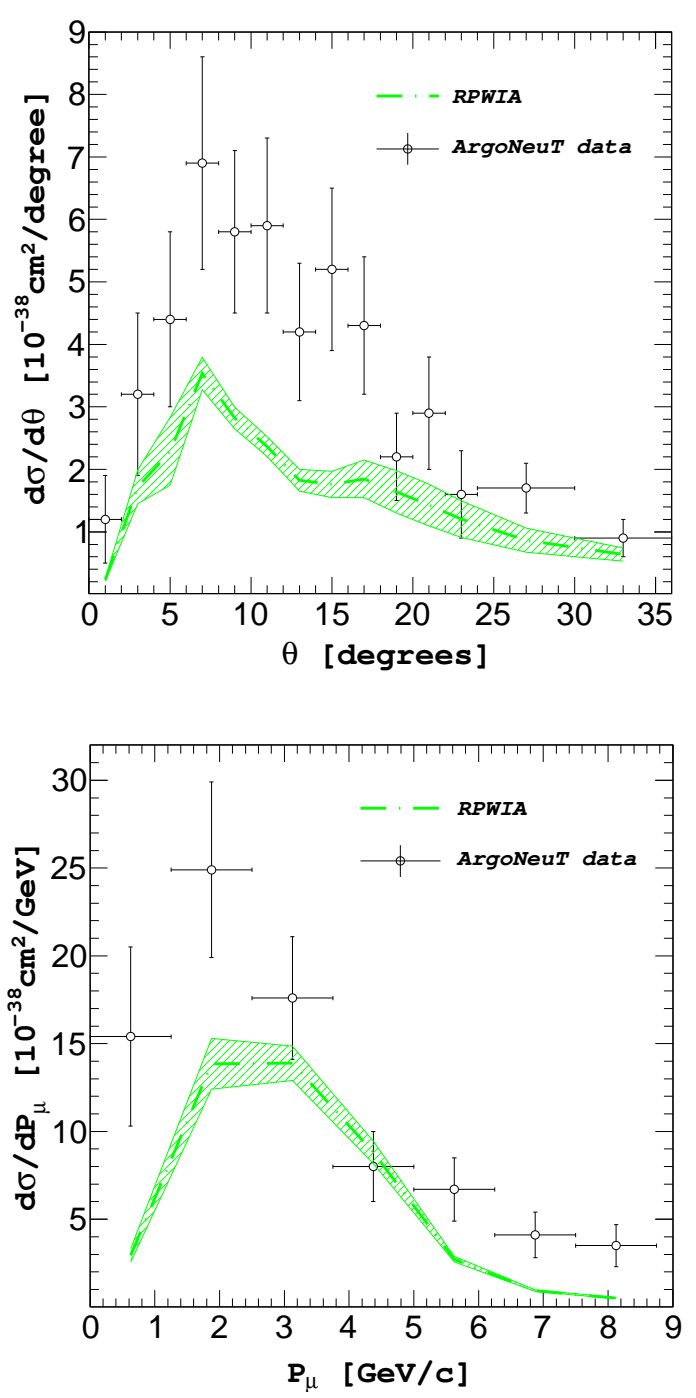

Figure 3. (Color online) Estimated uncertainties for the fluxaveraged differential cross sections $d \sigma / d \theta$ and $d \sigma / d P_{\mu}$ on ${ }^{40} \mathrm{Ar}$ calculated in RPWIA. The data are from ArgoNeuT [26].

are essentially due to the different imaginary part of the three potentials, which accounts for the overall effects of inelastic channels and is not univocally determined from the elastic phenomenology. In contrast, the real term is similar for different parameterizations and gives similar results. We observe that the DEM and UNDEM potentials give in general close results; in fact, even if they are obtained using very different fitting procedures 89], they are based on the same dataset of elastic-scattering data. In constrast, the EDAD1 potential produces somewhat smaller results and larger differences in comparison with the results of the DEM and UNDEM potentials: for instance, in the peak region with $\theta \approx 5-7 \mathrm{deg}$, the RGFDEM and RGF-EDAD1 cross sections differ by about $20 \%-30 \%$.

The RGF cross sections are generally larger than the RPWIA and the RMF ones, but they are in general sig- 

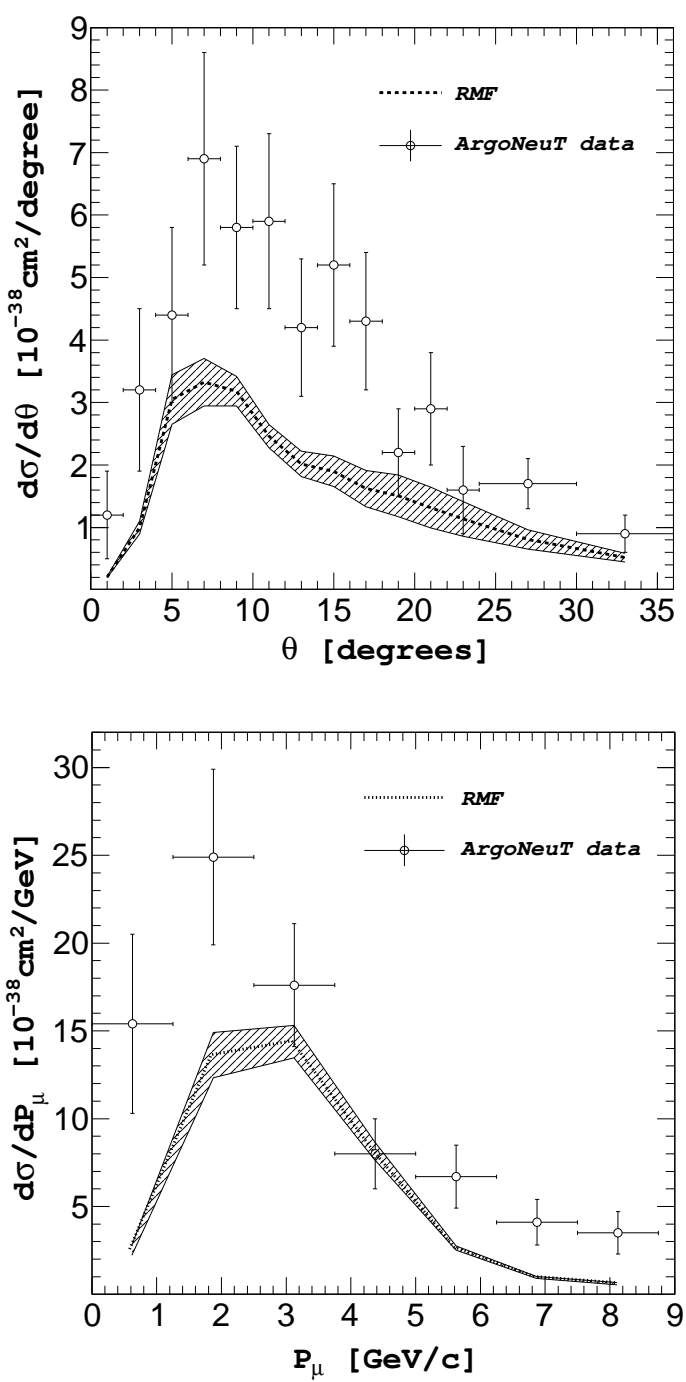

Figure 4. (Color online) The same as in Fig. 3 but in RMF.

nificantly lower than the data, but for $\theta \leq 5 \mathrm{deg}$ and $\theta \geq 20 \mathrm{deg}$. In the RGF the imaginary part of the optical potential redistributes the flux in all the final-state channels and, in each channel, the flux lost towards other channels is compensated by the flux gained from the other channels. The larger cross sections in the RGF arise from the translation to the inclusive strength of the overall effects of inelastic channels which are not included in the other models such as, for instance, rescattering processes of the nucleon in its way out of the nucleus, nonnucleonic $\Delta$ excitations which may arise during nucleon propagation, or also some multinucleon processes. These contributions are not included explicitly in the RGF, but they can be recovered, to some extent, by the imaginary part of the phenomenological OP.

We note that in all the calculations presented in this work we have used the standard value of the axial mass $M_{A}=1.03 \mathrm{GeV}$. A larger value of $M_{A}$ would increase the cross section and improve the agreement with data.
In Fig. 2 the CC differential cross section $d \sigma / d P_{\mu}$ integrated over the ArgoNeuT flux is shown as a function of the muon momentum $P_{\mu}$. Also in this case the RPWIA and the RMF results are lower than the experimental data, while the RGF produces larger cross sections which are in better agreement with the data. Anyway, the first two measurements in the low energy bins of $P_{\mu}$ are underestimated by all the results by a factor of 2 .

All the models which have been adopted for the present calculations are based on the IA, where the cross section is given by an incoherent sum of interactions between the incident neutrino and all the single nucleons of the nucleus. Also the RGF, which is a more complex model and, with the use of the complex OP, is able to recover contributions of channels which are not included in the other models, is essentially based on the IA. Models based on IA have been successful in the description of QE exclusive and inclusive electron scattering [38]. Moreover, the RGF is able to give a reasonable description of CCQE and NCE MiniBooNE cross sections [79 81]. In the inclusive CC neutrino-nucleus scattering at energies larger than a few GeVs, however, all these models may neglect important contributions of reaction processes which can be included in the experimental cross sections. It is therefore not surprising that the calculated cross sections in Figs. 1 and 2 are generally lower than the experimental data.

With the aim to give a more quantitative information we have tried to estimate the uncertainties of our calculations. The most obvious source of uncertainty is the neutrino flux: it is usually known with sufficient precision but its errors are not negligible [26]. For energies up to $10 \mathrm{GeV}$ the ArgoNeuT flux is given in bins of resolution of 1 or $2 \mathrm{GeV}$ and, for each bin, we compute an "average" cross section starting from five or more calculations at different energies. It is straightforward to assume that this procedure introduces additional uncertainties in our results. In Figs. 3 and 4 we show our results for the $d \sigma / d \theta$ and $d \sigma / d P_{\mu}$ differential cross section in RPWIA and in RMF. The bands in the figures represent these two errors added in quadrature. In the interval of $\theta$ and $P_{\mu}$ covered by the ArgoNeuT kinematics the two errors are generally small and neither the RPWIA nor the RMF cross sections can reproduce the experimental data within the error bands. This is a reasonable result since the RPWIA and RMF cross sections in each bin are stable and the uncertainties on the neutrino flux are generally small.

In the case of the RGF we consider two additional sources of errors. The RGF results presented here contain the contribution of both terms of the hadron tensor in Eq. (25) of [63]. The calculation of the second term, which is entirely due to the imaginary part of the OP, is a hard and time consuming numerical task which requires the integration over all the eigenfunctions of the continuum spectrum of the optical potential. Numerical uncertainties on this term are anyhow under control and, from many calculations in different kinematics, have been 

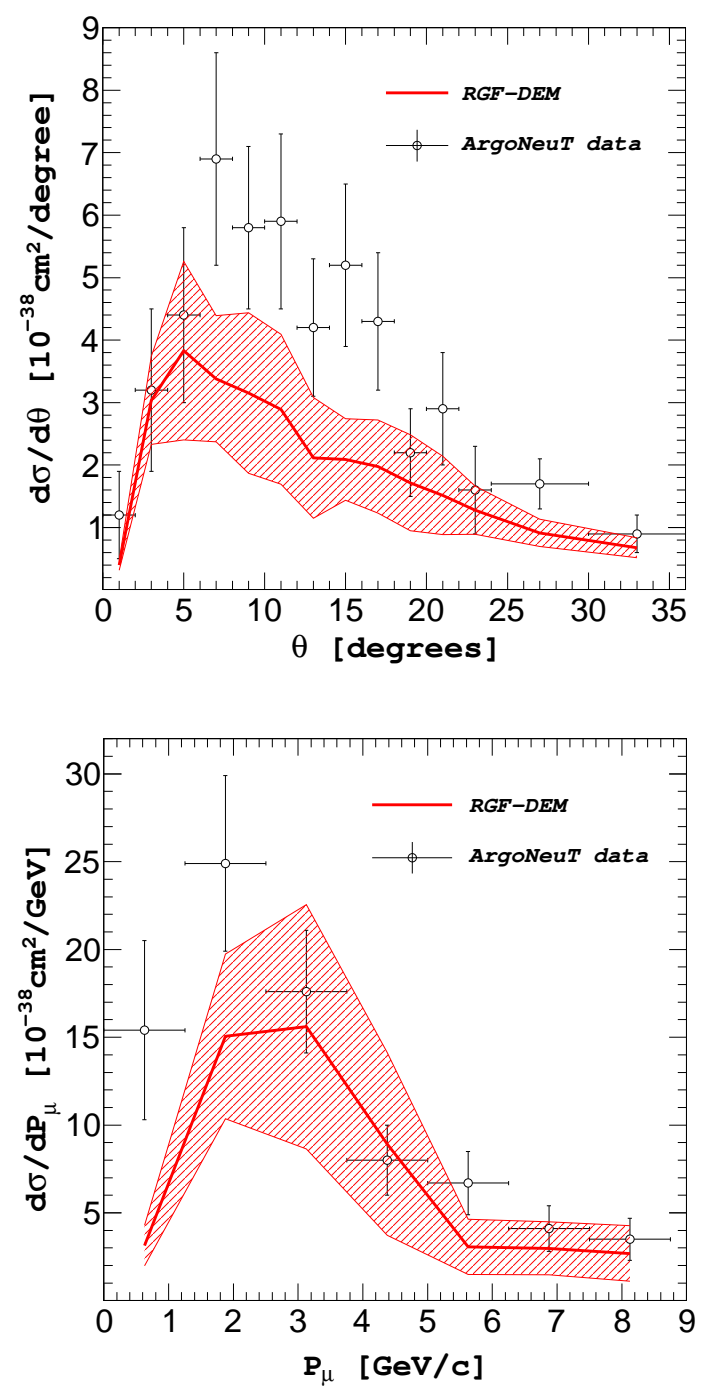

Figure 5. (Color online) The same as in Fig. 3 but in RGFDEM.

estimated at most within $10 \%$.

The fact that in actual RGF calculations we have to use a phenomenological energy dependent OP introduces additional complications. The OPs in [88, 89] have been tested for energies up to $\approx 1 \mathrm{GeV}$ and to provide results up to $\approx 10 \mathrm{GeV}$ we have to extend the range of validity of these parameterizations. This has been done multiplying each term of the OP by a realistic function of the energy that has been chosen to carefully reproduce the behavior of the OP around $1 \mathrm{GeV}$. We have checked that our results do not depend significantly on the multiplying function. The RGF-DEM $d \sigma / d \theta$ and $d \sigma / d P_{\mu}$ differential cross sections are shown in Fig. [5 where the error bands represent all the uncertainties that we have considered added in quadrature. Similar results with similar uncertainties are obtained in the case of RGF-UNDEM and RGF-EDAD1. The error bands for the RGF results are larger than for RPWIA and RMF and, as a consequence, the upper limits of the RGF cross sections are closer to the data. This outcome can be ascribed to the moderately large uncertainties on the cross sections in each experimental bin of neutrino energy, in particular for neutrino energies of $\approx 2-3 \mathrm{GeV}$ and small energy transferred to the nucleus.

The large error bands in Fig. 5 do not allow us to draw any definite conclusion. However, the results presented in this work indicate that the RGF, as well as the other models based on the IA, generally underpredict ArgoNeuT data, but they are able to reproduce the shape and the correct order of magnitude of the experimental cross section.

\section{SUMMARY AND CONCLUSIONS}

In this paper we have compared the predictions of different relativistic descriptions of FSI for CC neutrinonucleus scattering in the ArgoNeuT kinematics. In the RPWIA FSI are neglected; in the RMF they are described using the same relativistic mean field potential considered in describing the initial nucleon state; in the RGF the full complex OP, with its real and imaginary parts, is used to account for FSI. All final-state channels are included in the RGF, the flux lost in each channel is recovered in the other channels by the imaginary part of the OP making use of the dispersion relations and the total flux is conserved. The RGF gives a good description of the $\left(e, e^{\prime}\right)$ data in the $\mathrm{QE}$ region and it is also able to describe both CCQE and NCE MiniBooNE data. In the RGF cross sections the contribution of reaction channels that are neglected in the other models, e.g., rescattering processes of the nucleon in its way out of the nucleus, non-nucleonic $\Delta$ excitations, which may arise during nucleon propagation, with or without real pion production, or also multinucleon processes, is translated, to some extent, into the inclusive strength by the imaginary part of the phenomenological OP. However, the role of the various reaction channels included in the phenomenological $\mathrm{OP}$, cannot be disentangled and the enhancement of the cross section cannot be attributed to a specific process. In order to clarify this point, a careful evaluation of all nuclear effects and of the relevance of multinucleon emission and of some non-nucleonic contributions, as well as a better determination of the relativistic OP, which closely fulfills the dispersion relations, would reduce the theoretical uncertainties on the RGF.

Our results give a clear indication that IA-based models are able to reproduce the correct order of magnitude and the shape of the ArgoNeuT data but they generally underpredict the experimental cross sections, in particular for lower values of $P_{\mu}$ and for values of $\theta$ between 5 deg and 20 deg. A careful evaluation of all nuclear effects is required to recover some important contributions to the CC inclusive strenght. In particular, a careful study of medium effects in the few-GeV energy region that takes into account quasielastic, inelastic, as well as deep-inelastic processes, is highly desirable. 


\section{ACKNOWLEDGMENTS}

This work was partially supported by the Italian MIUR through the PRIN 2009 research project.

[1] K. Abe et al. (Super-Kamiokande Collaboration), Phys. Rev. D 83, 052010 (2011).

[2] K. Abe et al. (Super-Kamiokande Collaboration), Phys. Rev. Lett. 107, 241801 (2011).

[3] M. Antonello et al. (ICARUS Collaboration), Eur. Phys. J. C 73, 1 (2013).

[4] B. Aharmim et al. (SNO Collaboration), Phys. Rev. C 81, 055504 (2010).

[5] P. Adamson et al. (MINOS Collaboration), Phys. Rev. Lett. 107, 021801 (2010).

[6] K. Abe et al. (T2K Collaboration), Phys. Rev. Lett. 107, 041801 (2011).

[7] A. A. Aguilar-Arevalo et al. (MiniBooNE Collaboration), Phys. Rev. Lett. 110, 161801 (2013).

[8] K. B. M. Mahn et al. (MiniBooNE and SciBooNE Collaborations), Phys. Rev. D 85, 032007 (2012).

[9] G. Cheng et al. (MiniBooNE and SciBooNE Collaborations), Phys. Rev. D 86, 052009 (2012)

[10] Y. Abe et al. (Double Chooz Collaboration), Phys. Rev. Lett. 108, 131801 (2012).

[11] F. P. An et al. (Daya Bay Collaboration), Phys. Rev. Lett. 108, 171803 (2012).

[12] F. P. An et al. (Daya Bay Collaboration), Chin. Phys. C 37, 011001 (2013)

[13] J. K. Ahn et al. (RENO Collaboration), Phys. Rev. Lett. 108, 191802 (2012).

[14] M. H. Ahn, , et al. (K2K Collaboration), Phys. Rev. D 74, 072003 (2006).

[15] A. Aguilar et al. (LSND Collaboration), Phys. Rev. D 64, 112007 (2001).

[16] J. M. Conrad, C. M. Ignarra, G. Karagiorgi, M. H. Shaevitz, and J. Spitz, Adv. High Energy Phys. 2013, 163897 (2013).

[17] S. Petcov, Adv.High Energy Phys. 2013, 852987 (2013)

[18] E. Giusarma, M. Archidiacono, R. de Putter, A. Melchiorri, and O. Mena, Phys. Rev. D 85, 083522 (2012).

[19] G. Mention, M. Fechner, T. Lasserre, T. A. Mueller, D. Lhuillier, M. Cribier, and A. Letourneau, Phys. Rev. D 83, 073006 (2011).

[20] J. Hamann, S. Hannestad, G. G. Raffelt, and Y. Y. Y. Wong, Journal of Cosmology and Astroparticle Physics 2011, 03

[21] M. Sorel, J. M. Conrad, and M. H. Shaevitz, Phys. Rev. D 70, 073004 (2004).

[22] A. A. Aguilar-Arevalo et al. (MiniBooNE Collaboration), Phys. Rev. D 81, 092005 (2010).

[23] A. A. Aguilar-Arevalo et al. (MiniBooNE Collaboration), Phys. Rev. D 82, 092005 (2010).

[24] A. A. Aguilar-Arevalo et al. (MiniBooNE Collaboration), (2013), arXiv:1301.7067 [hep-ex].

[25] Y. Nakajima et al. (SciBooNE Collaboration), Phys. Rev. D 83, 012005 (2011).

[26] C. Anderson et al. (ArgoNeuT Collaboration), Phys. Rev. Lett. 108, 161802 (2012).

[27] MiniBooNE, http://www-boone.fnal.gov
[28] Minerva, http://minerva.fnal.gov/

[29] T2K, http://t2k-experiment.org/

[30] G. Karagiorgi, (2013), arXiv:1304.2083 [physics.ins-det]

[31] T. Suzuki and M. Honma, Phys. Rev. C 87, 014607 (2013).

[32] O. Benhar, P. Coletti, and D. Meloni, Phys. Rev. Lett. 105, 132301 (2010)

[33] O. Benhar and G. Veneziano, Phys. Lett. B 702, 433 (2011).

[34] A. V. Butkevich, Phys. Rev. C 82, 055501 (2010)

[35] A. V. Butkevich and D. Perevalov, Phys. Rev. C 84, 015501 (2011).

[36] C. Juszczak, J. T. Sobczyk, and J. Zmuda, Phys. Rev. C 82, 045502 (2010).

[37] S. Boffi, C. Giusti, and F. D. Pacati, Phys. Rept. 226, 1 (1993).

[38] S. Boffi, C. Giusti, F. D. Pacati, and M. Radici, Electromagnetic Response of Atomic Nuclei, Oxford Studies in Nuclear Physics, Vol. 20 (Clarendon Press, Oxford, 1996).

[39] M. Martini, M. Ericson, G. Chanfray, and J. Marteau, Phys. Rev. C 80, 065501 (2009).

[40] M. Martini, M. Ericson, G. Chanfray, and J. Marteau, Phys. Rev. C 81, 045502 (2010).

[41] M. Martini, M. Ericson, and G. Chanfray, Phys. Rev. C 84, 055502 (2011).

[42] J. Nieves, I. Ruiz Simo, and M. J. Vicente Vacas, Phys. Rev. C 83, 045501 (2011).

[43] J. Nieves, I. Ruiz Simo, and M. J. Vicente Vacas, Physics Letters B 707, 72 (2012)

[44] J. Nieves, I. Ruiz Simo, and M. J. Vicente Vacas, Physics Letters B 721, 90 (2013)

[45] J. E. Amaro, M. B. Barbaro, J. A. Caballero, T. W. Donnelly, and C. F. Williamson, Phys. Lett. B 696, 151 (2011).

[46] J. E. Amaro, M. B. Barbaro, J. A. Caballero, T. W. Donnelly, and J. M. Udías, Phys. Rev. D 84, 033004 (2011).

[47] J. E. Amaro, M. B. Barbaro, J. A. Caballero, and T. W. Donnelly, Phys. Rev. Lett. 108, 152501 (2012)

[48] A. Bodek, H. Budd, and M. Christy, Eur. Phys. J. C 71, 1 (2011)

(249]1)T. Golan, K. M. Graczyk, C. Juszczak, and J. T. Sobczyk, (2013), arXiv:1302.3890 [hep-ph].

[50] T. Leitner, O. Buss, L. Alvarez-Ruso, and U. Mosel, Phys. Rev. C 79, 034601 (2009).

[51] T. Leitner U. and Mosel, Phys. Rev. C 81, 064614 (2010).

[52] A. M. Ankowski and O. Benhar, Phys. Rev. C 83, 054616 (2011).

[53] E. Fernandez Martinez and D. Meloni, Physics Letters B 697, 477 (2011).

[54] J. M. Udías, P. Sarriguren, E. Moya de Guerra, E. Garrido, and J. A. Caballero, Phys. Rev. C 48, 2731 (1993).

[55] A. Meucci, C. Giusti, and F. D. Pacati, Phys. Rev. C 64, 014604 (2001). 
[56] A. Meucci, C. Giusti, and F. D. Pacati, Phys. Rev. C 64, 064615 (2001)

[57] A. Meucci, Phys. Rev. C 65, 044601 (2002)

[58] M. Radici, A. Meucci, and W. H. Dickhoff, Eur. Phys. J. A 17, 65 (2003).

[59] C. Giusti, A. Meucci, F. D. Pacati, G. Co', and V. De Donno, Phys. Rev. C 84, 024615 (2011)

[60] C. Maieron, M. C. Martinez, J. A. Caballero, and J. M. Udías, Phys. Rev. C 68, 048501 (2003)

[61] J. A. Caballero, Phys. Rev. C 74, 015502 (2006).

[62] J. A. Caballero, M. C. Martinez, J. L. Herraiz, and J. M. Udías, Phys. Lett. B 688, 250 (2010).

[63] A. Meucci, C. Giusti, and F. D. Pacati, Nuclear Physics A 739, 277 (2004).

[64] A. Meucci, F. Capuzzi, C. Giusti, and F. D. Pacati, Phys. Rev. C 67, 054601 (2003).

[65] A. Meucci, C. Giusti, and F. D. Pacati, Nuclear Physics A 744, 307 (2004)

[66] A. Meucci, C. Giusti, and F. D. Pacati, Acta Phys. Polon. B 37, 2279 (2006)

[67] A. Meucci, C. Giusti, and F. D. Pacati, Nuclear Physics A 773, 250 (2006)

[68] A. Meucci, C. Giusti, and F. D. Pacati, Phys. Rev. C 77, 034606 (2008).

[69] C. Giusti, A. Meucci, and F. D. Pacati, Acta Phys. Polon. B 40, 2579 (2009).

[70] A. Meucci, J. A. Caballero, C. Giusti, F. D. Pacati, and J. M. Udías, Phys. Rev. C 80, 024605 (2009).

[71] A. Meucci, J. A. Caballero, C. Giusti, and J. M. Udías, Phys. Rev. C 83, 064614 (2011).

[72] C. Giusti and A. Meucci, EPJ Web of Conferences 38, 14004 (2012).

[73] Y. Horikawa, F. Lenz, and N. C. Mukhopadhyay, Phys. Rev. C 22, 1680 (1980).

[74] F. Capuzzi, C. Giusti, and F. D. Pacati, Nuclear Physics A 524, 681 (1991).

[75] F. Capuzzi, C. Giusti, F. D. Pacati, and D. N. Kadrev,
Annals of Physics (N.Y.) 317, 492 (2005)

[76] A. Meucci, C. Giusti, and F. D. Pacati, Nuclear Physics A 756, 359 (2005).

[77] C. Giusti and A. Meucci, Journal of Physics: Conference Series 336, 012025 (2011).

[78] A. Meucci, M. Vorabbi, C. Giusti, F. D. Pacati, and P. Finelli, (2013), arXiv:1302.3390 [nucl-th],

[79] A. Meucci, M. B. Barbaro, J. A. Caballero, C. Giusti, and J. M. Udías, Phys. Rev. Lett. 107, 172501 (2011).

[80] A. Meucci and C. Giusti, Phys. Rev. D 85, 093002 (2012)

[81] A. Meucci, C. Giusti, and F. D. Pacati, Phys. Rev. D 84, 113003 (2011)

[82] B. D. Serot and J. D. Walecka, Adv. Nucl. Phys. 16, 1 (1986).

[83] P. Finelli, N. Kaiser, D. Vretenar, and W. Weise, Nuclear Physics A 735, 449 (2004).

[84] P. Finelli, N. Kaiser, W. Weise, and D. Vretenar, "Nuclear density functional constrained by low-energy QCD," in Theoretical Nuclear Physics In Italy, edited by S. Boffi, A. Covello, M. Di Toro, A. Fabrocini, G. Pisent, and S. Rosati (World Scientific, Singapore, 2004) Chap. 5, pp. 45-52.

[85] P. Finelli, N. Kaiser, D. Vretenar, and W. Weise, Nucl. Phys. A770, 1 (2006)

[86] G. Co', V. De Donno, P. Finelli, M. Grasso, M. Anguiano, A. M. Lallena, C. Giusti, A. Meucci, and F. D. Pacati, Phys. Rev. C 85, 024322 (2012).

[87] C. Giusti, A. Meucci, F. D. Pacati, G. Co', and V. De Donno, Journal of Physics: Conference Series 366, 012019 (2012)

[88] E. D. Cooper, S. Hama, B. C. Clark, and R. L. Mercer, Phys. Rev. C 47, 297 (1993).

[89] E. D. Cooper, S. Hama, and B. C. Clark, Phys. Rev. C 80, 034605 (2009). 